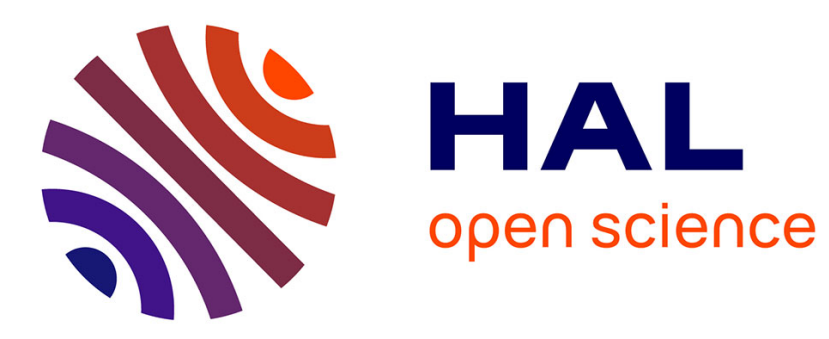

\title{
Convex particles at interfaces
}

Elie Raphaël, Jean-Marc Di Meglio, Marcel Berger, Eugenio Calabi

\section{To cite this version:}

Elie Raphaël, Jean-Marc Di Meglio, Marcel Berger, Eugenio Calabi. Convex particles at interfaces. Journal de Physique I, 1992, 2 (5), pp.571-579. 10.1051/jp1:1992112 . jpa-00246519

\section{HAL Id: jpa-00246519 https://hal.science/jpa-00246519}

Submitted on 1 Jan 1992

HAL is a multi-disciplinary open access archive for the deposit and dissemination of scientific research documents, whether they are published or not. The documents may come from teaching and research institutions in France or abroad, or from public or private research centers.
L'archive ouverte pluridisciplinaire HAL, est destinée au dépôt et à la diffusion de documents scientifiques de niveau recherche, publiés ou non, émanant des établissements d'enseignement et de recherche français ou étrangers, des laboratoires publics ou privés. 
Classification

Physics Abstracts

$68.10 \mathrm{C}-02.40$

\title{
Convex particles at interfaces
}

Elie Raphaël ( $\left.{ }^{1}\right)$, Jean-Marc di Meglio ( $\left.{ }^{1}\right)$, Marcel Berger $\left({ }^{2}\right)$ and Eugenio Calabi ( $\left.{ }^{3}\right)$

(1) Laboratoire de Physique de la Matière Condensée, Collège de France, 11 place MarcelinBerthelot, 75231 Paris Cedex 05, France

${ }^{2}$ ) Institut des Hautes Etudes Scientifiques, 35 route de Chartres, 91440 Bures sur Yvette, France

( ${ }^{3}$ ) Department of Mathematics, University of Pennsylvania, Philadelphia, PA 19104-6395 U.S.A.

(Received 29 August 1991, revised 30 January 1992, accepted 7 February 1992)

\begin{abstract}
We study the equilibrium positions of a long prismatic particle of convex crosssection at a liquid/liquid interface in the gravity-free situation. We show in particular that : i) in a stable equilibrium position the contact angles at both three-phase lines obey the Young equation, ii) for any convex cross-section there are at least four positions for which contact angles obey the Young equation. Moreover, the stability of these positions is investigated.
\end{abstract}

\section{Equilibrium position.}

In the gravity-free situation, a spherical particle 3 will be embedded in a planar [1] interface between a liquid 1 and a liquid 2 provided that the following condition is fulfilled [2] :

$$
\left|\frac{\gamma_{13}-\gamma_{23}}{\gamma_{12}}\right|<1
$$

(where the $\gamma_{i j}$ 's are the interfacial tensions characterizing the system). The position of the particle at the interface then is such that the contact angle between the tangents of surfaces 1-2 and 2-3 at the three-phase line is the Young angle $\theta_{\mathrm{y}}$ defined by the Young equation (Fig. 1) :

$$
\cos \theta_{y}=\frac{\gamma_{13}-\gamma_{23}}{\gamma_{12}}
$$

Our aim is to understand how these specific properties of spherical particles may be generalized to particles of arbitrary shape. For sake of simplicity, we consider long prismatic particles of convex cross-section (Fig. 2). The problem then amounts to a purely 2-

(*) URA 792 du CNRS. 


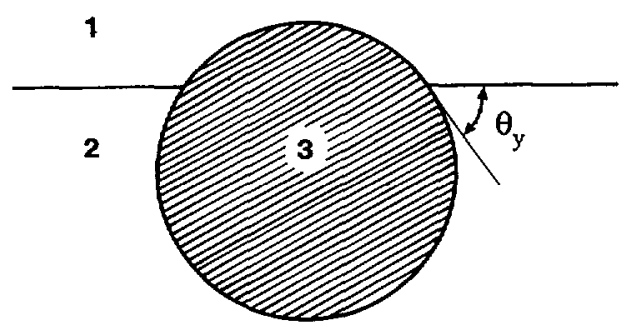

Fig. 1. - A spherical particle at a liquid-liquid interface.

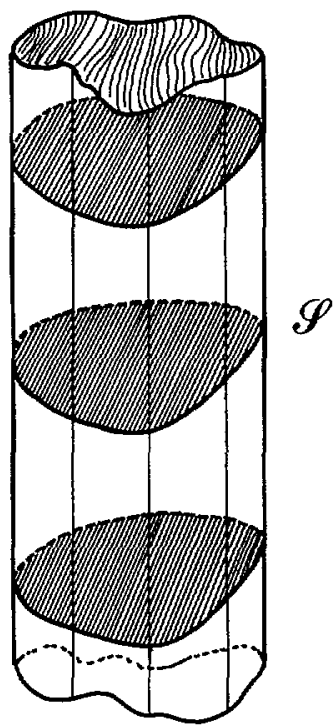

Fig. 2. - A prismatic particle with a convex cross-section $\delta$. We study the equilibrium of this particle at a liquid-liquid interface, the generating lines being parallel to the interface.

dimensional problem whose geometry is pictured in figure 3. The cross-section of the solid is a plane region bounded by a simple, closed, convex curve $S$; we shall assume henceforth, in addition, that $S$ is smooth of class at least $C^{3}$ and has strictly positive curvature (i.e. finite radius of curvature) everywhere. Since we assume that there are no gravity effects, the liquidfluid interface is flat and represented by a straight line $y=a x+b$ in the plane of the figure ; it intercepts $S$ at two points $\left(x_{1}, y_{1}\right)$ and $\left(x_{2}, y_{2}\right)$.

The energy $W$ of the system is given by :

$$
W=\gamma_{13} \ell_{13}+\gamma_{23} \ell_{23}-\gamma_{12} \ell_{12}
$$

where $\ell_{13}$ and $\ell_{23}$ are the length of contact between the solid and the liquids and $\ell_{12}$ is the length of the intercept of the liquid-liquid interface by the solid. $W$ is a function of both variables $a$ and $b$. The solid particle is in a stable equilibrium at the interface when $W$ is minimum, which implies :

$$
\frac{\partial W}{\partial a}=\frac{\partial W}{\partial b}=0
$$




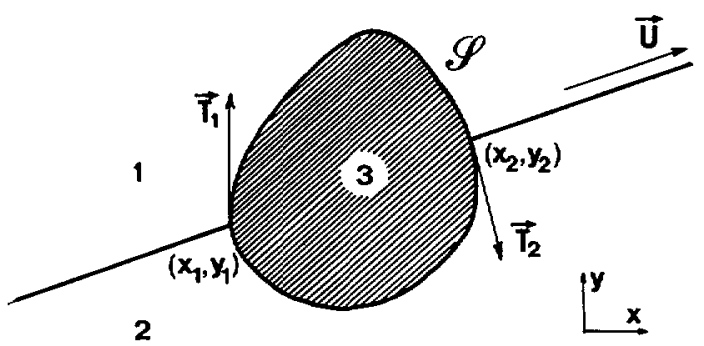

Fig. 3. - Convex prismatic particle trapped at a liquid-liquid interface. We rotate the interface (Eq. : $y=a x+b$ ) while keeping the solid fixed.

Note that condition (4) is necessary but not sufficient to ensure a stable equilibrium position : we come back to this point in section 3 . From (3) and (4) one easily obtains :

$$
\mathbf{T}_{1} \cdot \mathbf{U}=\mathbf{T}_{2} \cdot \mathbf{U}=\frac{\boldsymbol{\gamma}_{13}-\gamma_{23}}{\gamma_{12}}
$$

where $\mathbf{T}_{1}$ and $\mathbf{T}_{2}$ are the unitary vectors tangent to the solid $\delta$ at the triple points $\left(x_{1}, y_{1}\right)$ and $\left(x_{2}, y_{2}\right)$ (Fig. 3$) ; \mathbf{U}$ is the unitary vector carried by the liquid/liquid interface. Equation (5) is meaningful only when condition (1) is satisfied ; we finally have (using (2))

$$
\mathbf{T}_{1} \cdot \mathbf{U}=\mathbf{T}_{2} \cdot \mathbf{U}=\cos \theta_{\mathrm{y}} .
$$

We therefore conclude that if a convex prismatic particle is trapped in a stable equilibrium position at a liquid/liquid interface, then the Young relation is fulfilled at both triple points, as expected from a local force balance.

\section{Existence of Young positions.}

We now address the following problem : for a given Young angle $\theta_{\mathrm{y}}$ and a given convex curve $\delta$, is it always possible to find a position which satisfies conditions (6) ? (Such a position will hereafter be called a « Young position ».) The answer may be obtained by studying the construction of figure 4. We consider a 2-dimensional cone with an angle $\pi-2 \theta_{y}$ (at the

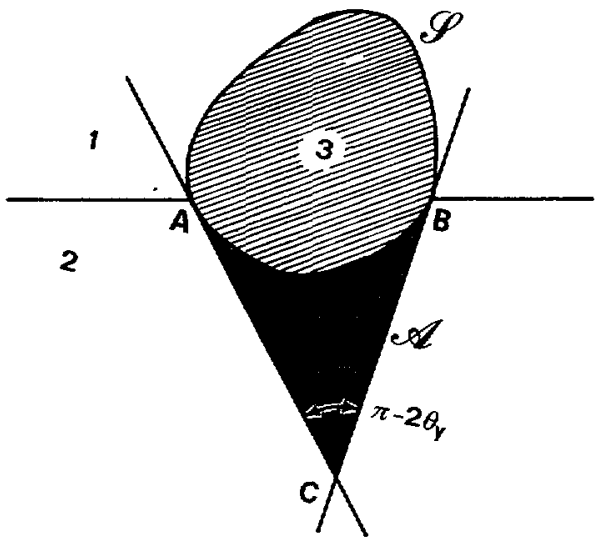

Fig. 4. - Geometrical construction used to derive the existence of Young positions. 
vertex $C$ ). The convex curve $S$ is then positioned inside the cone as indicated. Let $A$ and $B$ be the points where the cone and $S$ are tangent and $A$ the area delimited by the triangle $(A, B, C)$ and the curve $S$ (shaded area). Since after a complete rotation of $S$ inside the cone the area $A$ recovers its initial value, it has at least two extrema. We will now show that when $A$ is extremal, the triangle ( $A, B, C$ ) is isosceles (the angles in $A$ and $B$ being equal). Let us consider a position where $\mathcal{A}$ is extremum and rotate the curve $S$ (or equivalently the cone) by an infinitesimally small angle $\alpha$ (Fig. 5). Since $A$ is extremal the hatched areas must be equal, which yields (to the first order in $\alpha$ ) :

$$
\frac{1}{2} \alpha d_{\mathrm{AC}}^{2}=\frac{1}{2} \alpha d_{\mathrm{BC}}^{2}
$$

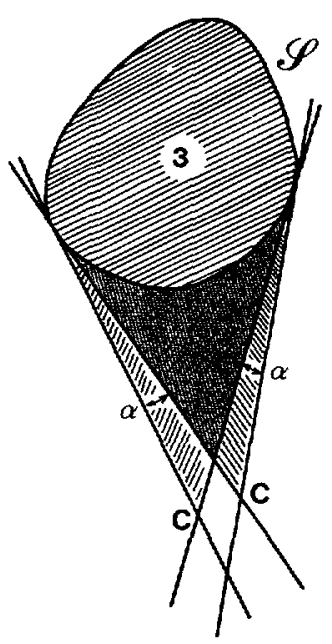

Fig. 5. - A small rotation (by an angle $\alpha$ ) of the cone starting from a position corresponding to an extremum of $\mathfrak{A}$.

or

$$
d_{\mathrm{AC}}=d_{\mathrm{BC}}
$$

(where $d_{\mathrm{AC}}$ is the distance between $\mathrm{A}$ and $\mathrm{C}$, etc.).

The triangle $(A, B, C)$ is thus isosceles. Since by construction the angle opposite side AB is $\pi-2 \theta_{\mathrm{y}}$, conditions (6) are satisfied at points A and B. We therefore conclude that positions for which the area $\mathcal{A}$ is extremal are Young positions (and vice versa). Since the area $\mathcal{A}$ has at least two extrema, there are at least two Young positions.

We will now refine the counting of the Young positions and show that in fact there are at least four such positions. We represent each point of the Euclidean plane by complex numbers $z=x+i y$, where $(x, y)$ are the Cartesian rectangular coordinates ; accordingly, we orient the plane by specifying that positive angles run counterclockwise. It is convenient to parametrize $s$ by the direction angle $\theta$ of the outward, unit normal vector, so that the unit tangent vector to $S$ with a positive orientation is represented by the complex variable $i \mathrm{e}^{i \theta}$, and $-\mathrm{e}^{i \theta}$ is the unit inward normal. We introduce the support function $u(\theta)=$ $\operatorname{Re}\left(\mathrm{e}^{-i \theta} z(\theta)\right)$, whose value for each $\theta$ expresses the oriented distance of the origin from the tangent line to $S$ at the point represented by $\theta$. Since the unit tangent vector to $S$ in the 
counterclockwise direction at $z(\theta)$ is represented by $i \mathrm{e}^{i \theta}$, the tangent line itself, denoted by $T_{\theta}$ is described by the implicit equation :

$$
0=\operatorname{Re}\left(\mathrm{e}^{-i \theta}(z-z(\theta))\right)=\operatorname{Re}\left(z \mathrm{e}^{-i \theta}\right)-u(\theta)
$$

thus $\mathrm{S}$ is uniquely determined by the support function $u(\theta)$, since it is the envelope of the oneparameter family of lines $T_{\theta}$ defined by (9). Conversely, given any smooth, real valued function $u(\theta)$ of $\theta$, periodic with period $2 \pi$, the envelope of the family of lines defined by (9) is the curve represented parametrically by $z(\theta)$, where

$$
z(\theta)=\mathrm{e}^{i \theta}\left(u(\theta)+i u^{\prime}(\theta)\right)
$$

the latter is a regular, smooth, convex curve, oriented counterclockwise with respect to increasing $\theta$, if and only if $u(\theta)$ satisfies everywhere the inequality

$$
\left|\frac{\mathrm{d} z}{\mathrm{~d} \theta}\right|=u^{\prime \prime}(\theta)+u(\theta)>0
$$

The function $u^{\prime \prime}(\theta)+u(\theta)$ represents at the same time the radius of curvature $R(\theta)$ of the resulting curve.

Given the Young angle $\theta_{y}$, we shall associate to each value of $\theta$ the two points $z\left(\theta+\theta_{\mathrm{y}}\right)$ and $z\left(\theta-\theta_{\mathrm{y}}\right)$ of $\mathcal{S}$. From these two points, we draw half-lines, respectively $\mathrm{L}_{+}$and $\mathrm{L}_{-}$, outward from $\mathrm{S}$, and parallel to the tangent line at $z(\theta):$ the half-lines, parametrized by a real $s \geqslant 0$, are given respectively by (Fig. 6) :

$$
L_{+} \cdot z=z\left(\theta+\theta_{\mathrm{y}}\right)+i s \mathrm{e}^{i \theta} ; \quad \mathbf{L}_{-} \quad z=z\left(\theta-\theta_{\mathrm{y}}\right)-i s \mathrm{e}^{i \theta}
$$

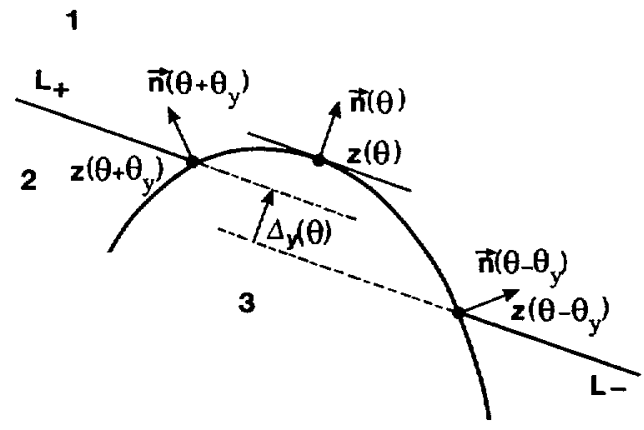

Fig. 6. - Definition of $\mathrm{L}_{+}, \mathrm{L}_{-}$and $\Delta_{\mathrm{y}}(\theta)\left(\theta_{\mathrm{y}}=45^{\circ}\right)$.

The distance between the parallel lines containing $L_{+}$and $L_{-}$is represented by the function :

$$
\begin{aligned}
\Delta_{\mathrm{y}}(\theta) & =\operatorname{Re}\left(\mathrm{e}^{-i \theta} z\left(\theta+\theta_{\mathrm{y}}\right)-\mathrm{e}^{-i \theta} z\left(\theta-\theta_{\mathrm{y}}\right)\right) \\
& =\cos \theta_{\mathrm{y}}\left(u\left(\theta+\theta_{\mathrm{y}}\right)-u\left(\theta-\theta_{\mathrm{y}}\right)\right)-\sin \theta_{\mathrm{y}}\left(u^{\prime}\left(\theta+\theta_{\mathrm{y}}\right)+u^{\prime}\left(\theta-\theta_{\mathrm{y}}\right)\right) .
\end{aligned}
$$

It is clear from figure 6 that any $\theta$ for which $\Delta_{y}(\theta)=0$ corresponds to a Young position.

For any given Young angle $\theta_{\mathrm{y}}$ and any convex curve $\delta$, we now associate a convex curve $\hat{\boldsymbol{s}}=\hat{\mathcal{S}}_{\theta_{\mathrm{y}}}$ defined by a corresponding support function $\hat{u}=\hat{u}_{\theta_{\mathrm{y}}}(\theta)$, obtained from $u(\theta)$ by the 
following convolution :

$$
\hat{u}(\theta)=\frac{\int_{-\theta_{y}}^{\theta_{y}}\left(\cos \phi-\cos \theta_{y}\right) u(\theta-\phi) \mathrm{d} \phi}{\int_{-\theta_{\mathrm{y}}}^{\theta_{\mathrm{y}}}\left(\cos \phi-\cos \theta_{\mathrm{y}}\right) \mathrm{d} \phi}
$$

The transformation of periodic functions $u(\theta)$ to other such functions $\hat{u}(\theta)$ has several elementary properties which are listed in appendix. We readily verify from (13) by taking partial derivatives with respect to the parameter $\theta_{\mathrm{y}}$ that $\Delta_{\mathrm{y}}(\theta)$ has the following integral representation :

$$
\begin{aligned}
\Delta_{\mathrm{y}}(\theta) & =-\int_{0}^{\theta_{\mathrm{y}}} \sin \eta\left\{u(\theta+\eta)-u(\theta-\eta)+u^{\prime \prime}(\theta+\eta)-u^{\prime \prime}(\theta-\eta)\right\} \mathrm{d} \eta \\
& =-\left(\hat{u}^{\prime \prime \prime}(\theta)+\hat{u}^{\prime}(\theta)\right) \int_{-\theta_{\mathrm{y}}}^{\theta_{y}}\left(\cos \phi-\cos \theta_{\mathrm{y}}\right) \mathrm{d} \phi .
\end{aligned}
$$

The last expression shows that $\theta$ corresponds to a Young position for the solid determined by the curve $\&$ (i.e. $\Delta_{\mathrm{y}}(\theta)=0$ ), if and only if the derivative of the radius of curvature $\hat{R}(\theta)=\hat{u}(\theta)+\hat{u}^{\prime \prime}(\theta)$ for the transformed curve $S$ is zero, meaning that $\theta$ corresponds to a vertex of $\hat{\delta}$. According to the four-vertex theorem [3], the curve $\hat{s}$ has at least four vertices and $S$ has therefore at least four Young positions. In the case where $\theta_{y} \rightarrow 0^{+}$, the transformed curve $\hat{\mathcal{s}}$, whose support function $\hat{u}$ at each $\theta$ is a weighted average of $u$ in the interval $\left[\theta-\theta_{\mathrm{y}}, \theta+\theta_{\mathrm{y}}\right]$ approaches uniformly $u$ itself, explaining the similarity between the above result and the classical four vertex theorem.

Figure 7 represents an ellipse and the four corresponding Young positions $\left(\theta_{\mathrm{y}}=\right.$ $45^{\circ}$ ) and figure 8 does the same for a less symmetric curve.

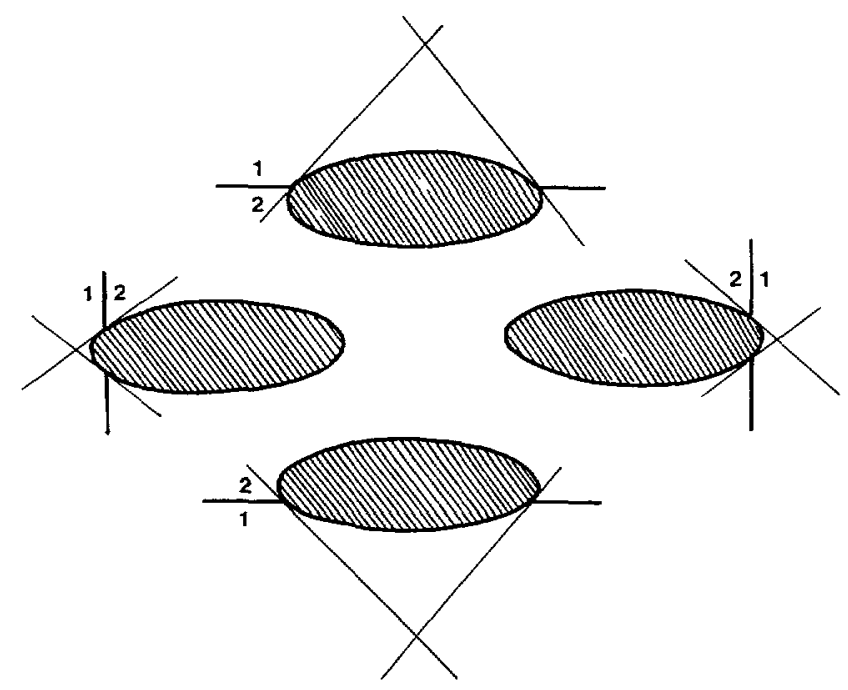

Fig. 7. - The four Young positions of an ellipse $\left(\theta_{y}=45^{\circ}\right)$. 

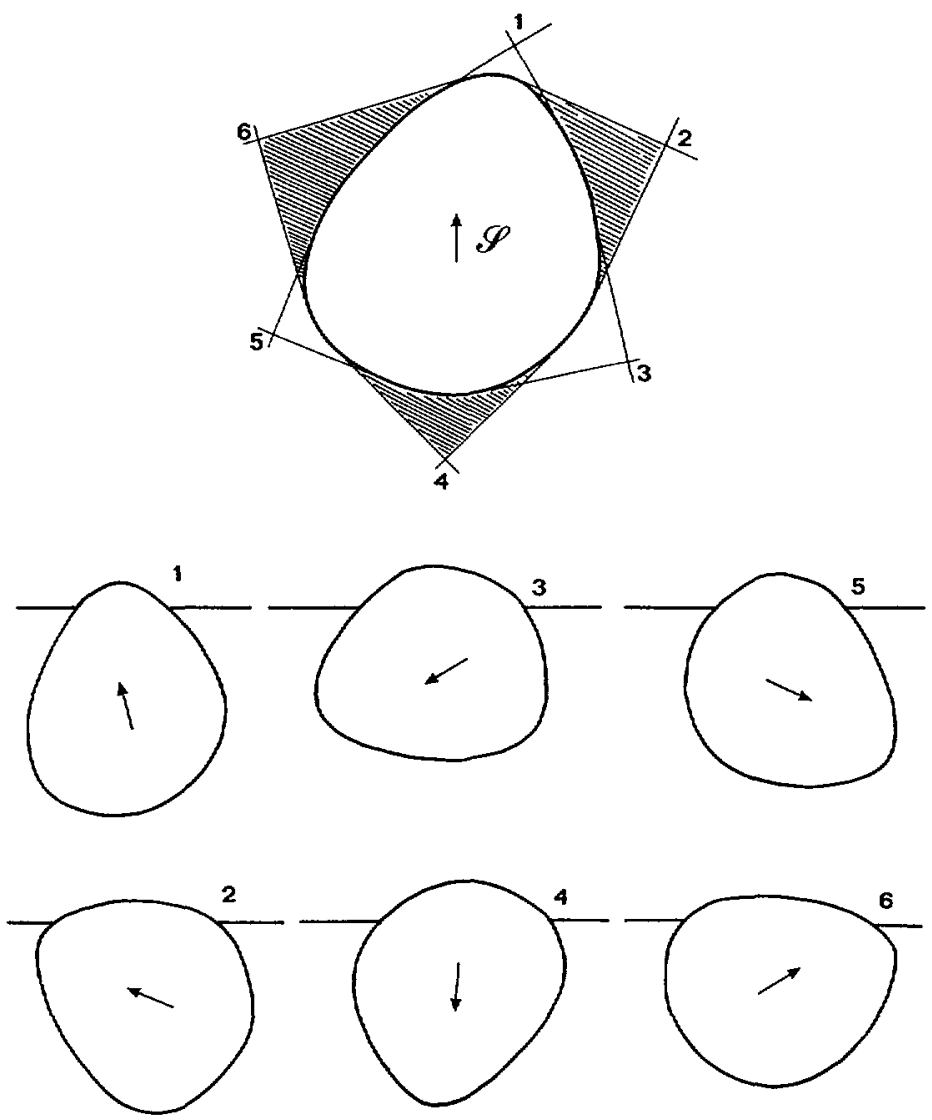

Fig. 8. - A convex cross-section $\delta$ with six Young positions $\left(\theta_{y}=45^{\circ}\right)$.

\section{Stability of Young positions.}

From section 1 we know that the first derivatives of $W$ are equal to zero for a Young position. But this is of course not enough to ensure that the position is stable : it may correspond to a maximum or to a saddle point of the energy. Let us imagine that, starting from a Young position we rotate (clockwise) the tangent in A by an angle $\alpha$ and the tangent in B by an angle $\beta$ ( $\alpha$ and $\beta$ being infinitesimally small); the corresponding change $\delta W$ in the system energy is then :

$$
\delta W=\frac{1}{2} \gamma_{12} \sin \theta_{\mathrm{y}}\left(\left(\alpha^{2} R_{\mathrm{A}}+\beta^{2} R_{\mathrm{B}}\right)-\frac{\sin \theta_{\mathrm{y}}}{d_{\mathrm{AB}}}\left(\alpha R_{\mathrm{A}}+\beta R_{\mathrm{B}}\right)^{2}\right)
$$

where $R_{\mathrm{A}}$ and $R_{\mathrm{B}}$ are the radii of curvature in $\mathrm{A}$ and $\mathrm{B}$ and $d_{\mathrm{AB}}$ the distance between $\mathrm{A}$ and $\mathrm{B}$. The determinant $D$ associated to (16) is given by :

$$
D=\left|\begin{array}{cc}
\frac{\partial^{2}(\delta W)}{\partial \alpha^{2}} & \frac{\partial^{2}(\delta W)}{\partial \alpha \partial \beta} \\
\frac{\partial^{2}(\delta W)}{\partial \beta \partial \alpha} & \frac{\partial^{2}(\delta W)}{\partial \beta^{2}}
\end{array}\right|=\gamma_{12}^{2} \sin ^{2} \theta_{\mathrm{y}} R_{\mathrm{A}} R_{\mathrm{B}}\left(1-\frac{\sin \theta_{\mathrm{y}}}{d_{\mathrm{AB}}}\left(R_{\mathrm{A}}+R_{\mathrm{B}}\right)\right)
$$


When $\alpha=\beta$ (that is when one considers the construction of Fig. 5), it is possible to relate the variation $\delta \mathcal{A}$ of the area to both $\delta W$ and $D$. A straightforward but tedious calculation gives :

$$
\delta \mathfrak{A}=-\frac{1}{4} \frac{\alpha^{2} d_{\mathrm{AB}}^{2}}{\cos \theta_{\mathrm{y}} \sin \theta_{\mathrm{y}}}\left(1-\frac{\sin \theta_{\mathrm{y}}}{d_{\mathrm{AB}}}\left(R_{\mathrm{A}}+R_{\mathrm{B}}\right)\right)
$$

i.e.

$$
\begin{aligned}
\delta \mathcal{A} & =-\frac{1}{2} \frac{d_{\mathrm{AB}}^{2}}{\gamma_{12} \cos \theta_{\mathrm{y}} \sin ^{2} \theta_{\mathrm{y}}\left(R_{\mathrm{A}}+R_{\mathrm{B}}\right)} \delta W_{\alpha=\beta} \\
& =-\frac{1}{4} \frac{\alpha^{2} d \underset{\mathrm{AB}}{2}}{\gamma_{12}^{2} R_{\mathrm{A}} R_{\mathrm{B}} \cos \theta_{\mathrm{y}} \sin ^{3} \theta_{\mathrm{y}}} D .
\end{aligned}
$$

When $\delta \mathcal{A}$ is negative both $\delta W_{\alpha=\beta}$ and $D$ are positive : maxima of $\mathcal{A}$ thus correspond to stable solutions. Conversely, when $\delta \mathcal{A}$ is positive, $D$ is negative : minima of $A$ thus correspond to saddle points of the energy. We then conclude that if there are only finitely many Young positions, generically the half of them which corresponds to maxima of $A$ are stable.

This result may also be derived by considering the function $\Delta_{\mathrm{y}}(\theta)$ defined in section 2 by (13). Indeed, if $\Delta_{y}(\theta) \neq 0$, then the sign of $\Delta_{y}(\theta)$ tells us which way the net surface tension forces, pulling $S$ along the directed half-lines $\mathrm{L}_{+}$and $\mathrm{L}_{-}$exert a torque [4]. Hence a Young position where $\Delta_{y}(\theta)=0$, is stable or unstable according to whether locally the function $\Delta_{\mathrm{y}}(\theta)$ is, respectively, increasing or decreasing.

\section{Conclusion.}

We have shown that for any smooth, strictly convex curve $S$ (of at least class $C^{3}$ ) there are at least four positions satisfying the Young conditions. Among these positions we have precised the half of them which corresponds to a stable equilibrium. Therefore, in the gravity-free situation, a long prismatic particle of convex cross-section $\delta$ can always be trapped at a liquidliquid interface (provided that (Eq. (1)) is satisfied). The validity of this result for a 3dimensional, non prismatic, convex solid is an open problem of practical importance.

\section{Acknowledgments.}

We thank P.-G. de Gennes who initiated this study and M. Veyssié for her constant encouragement.

\section{Appendix.}

The transformation (14) has several elementary properties :

i) It is a linear map ; positive valued functions yield positive valued functions.

ii) It commutes with the translation operator $u(\theta) \rightarrow u_{\alpha}(\theta)=u(\theta-\alpha)$ for any real constant $\alpha$; consequently it commutes with any linear differential operator with constant coefficients. In particular, if $v(\theta)=u(\theta)+u^{\prime \prime}(\theta)>0$ then also $\hat{v}(\theta)=\hat{u}(\theta)+\hat{u}^{\prime \prime}(\theta)>0$.

iii) The value of $\hat{u}(\theta)$ for each $\theta$ is a weighted average of values of the function $u$ for the variable distributed in the interval $\left[\theta-\theta_{\mathrm{y}}, \theta+\theta_{\mathrm{y}}\right]$; in particular if $u(\theta)$ is continuous, the uniform limit of $\hat{u}(\theta)$ as $\theta_{\mathrm{y}} \rightarrow 0^{+}$is $u(\theta)$.

iv) The inverse transform yielding $u(\theta)$ from $\hat{u}(\theta)$, can be obtained by Fourier series methods : this shows in particular that $\hat{u}(\theta)$ is continuously differentiable at least one time more than $u(\theta)$. 


\section{References}

[1] The case of a curved interface has been analyzed recently by DI MEGLIO J.-M. and RAPHAËL E., $J$. Colloid Interface Sci. 136 (1990) 581.

[2] Von REInders W., Kolloid-Z. 13 (1913) 235. A general reference is AdAMson A. W., Physical Chemistry of surfaces, 2nd Ed., Interscience Publishers (John Wiley \& Sons, New York, 1967).

[3] MukhopadhaYa S., Bull. Calcutta Math. Soc. 1 (1909) 31.

[4] Note that the configuration space considered here is different from the one used throughout this paper: up to now, we assumed that the two half-lines defining the liquid-liquid interface where included in the same direction. 